\title{
Meta-analysis Comparing the Transradial and Transfemoral Approaches for Percutaneous Coronary Intervention in Elderly Patients
}

\section{Waleed E Ali ${ }^{1,2 *}$, Amol A Bahekar ${ }^{2}$ and Sylvester U Ejah ${ }^{1,2}$}

${ }^{1}$ Department of Internal Medicine, Cape Fear Valley Medical Center, Affiliated with Campbell University School of Osteopathic Medicine, North Carolina, USA ${ }^{2}$ Department of Medicine and Cardiology, Cape Fear Valley Medical Center, North Carolina, USA

\begin{abstract}
Background: The transradial (TR) approach in percutaneous coronary intervention (PCl) has become popular among cardiologists, with potential advantages over the transfemoral (TF) approach demonstrated in previous studies conducted in unselected patients. Since elderly patients requiring $\mathrm{PCl}$ are a growing population, we aimed to compare the clinical outcomes of the 2 approaches in elderly population by conducting a meta-analysis of pooled data from randomized clinical trials and observational studies.
\end{abstract}

Methods: PubMed, Google Scholar, Medline, and Cochrane Library, were searched for studies comparing the TR and TF approaches in $\mathrm{PCl}$ among elderly patients.

Results: Thirteen studies met our inclusion criteria (4,066 patients: 2,540, TR; 1,526, TF). Pooled analysis demonstrated non-inferiority of the TR compared to the TF approach with regard to major adverse cardiovascular events (MACE) (odds ratio [OR] 0.64, 95\% confidence interval [Cl] 0.40-1.02, $\mathrm{P}=0.06$ ), cerebrovascular accidents (OR 1.82, 95\% Cl 0.28-11.68, $\mathrm{P}=0.53$ ), revascularization (OR 1.14, 95\% $\mathrm{Cl} 0.43-3.04, \mathrm{P}=0.79$ ), pseudoaneurysm formation (OR $0.33,95 \% \mathrm{Cl} 0.08-1.32, \mathrm{P}=0.12$ ), volume of contrast given (mean difference [MD] $-1.11,95 \% \mathrm{Cl}$ $-19.57-17.35, \mathrm{P}=0.91$ ), and procedure time (MD 0.40, 95\% Cl -3.62-4.42, $\mathrm{P}=0.84$ ). Major bleeding and ambulation time were lower in the TR group (OR 0.49, 95\% Cl 0.33-0.71, P=0.0002 and MD -10.51, 95\% Cl -19.33- -1.69, $\mathrm{P}=0.02$, respectively). However, the TF group showed a higher success rate (OR 1.71, 95\% Cl 1.09-2.67, $\mathrm{P}=0.02$ ).

Conclusion: The safety profiles of the TR and TF approaches of PCl in elderly patients were comparable; The TR approach was associated with earlier ambulation and lower risk of major bleeding.

Keywords: Transradial approach; Percutaneous coronary intervention; Transfemoral; Elderly

\section{Introduction}

Historically, the first transradial (TR) attempt at aortic imaging was published in 1948. It was then adopted by Campeau L. for coronary artery intervention (percutaneous coronary intervention or PCI) in 1989. This approach of PCI thereafter became a widespread practice among cardiologists in younger patients, given its potential advantages over the transfemoral (TF) technique, as shown in previously published studies [1-3]. PCI, in general, carries potential risks of bleeding and vascular access complications, owing to the use of antiplatelet medications and anticoagulants $[4,5]$. Elderly patients are prone to have such increased risks, due to their multiple co-morbidities and widespread peripheral vascular diseases, which pose a therapeutic challenge in this age group [6]. Although the TR procedure was encouraged in previous studies conducted in unselected patients with improved patient outcomes, the data is limited in the growing elderly population. We therefore conducted this meta-analysis involving up-to-date published data to compare the safety profile and clinical outcomes between the 2 approaches.

\section{Research Methodology}

The flow diagram for the meta-analysis is shown in Figure 1. The study was based on previously published articles; therefore, no ethics approval or patient consent was needed. We used 5 databases for our research to collect appropriate studies. PubMed, Google Scholar, reference lists of relevant articles, Cochrane Library, and Medline were the primary tools used in our study. A total of 2 investigators, including the author, were involved in identifying the required studies. Steps were taken to prevent duplication. The following terms were used in our research "transradial versus transfemoral", "percutaneous coronary artery intervention", elderly patients". Studies were included if they (1) compared the TR with the TF approaches in PCI; (2) targeted elderly patients $\geq 65$ years old; and (3) were randomized, as well as non-randomized, trials. Studies were excluded if (1) clinical outcomes were difficult to extract; and (2) the studies were observational without comparative outcomes. Written articles that were not in English were explored with the help of a translator if not accompanied by an English version, and none met our inclusion criteria. The quality of the randomized studies was assessed by the Jadad Score, which ranges from 0-5 [7]. Meanwhile, the quality of the observational studies was evaluated based on the Newcastle-Ottawa Scale (NOS) form [8]. A minimum score of 7 was considered a high-quality study. The comparative outcomes included major adverse cardiovascular events (MACE), cerebrovascular accidents, revascularization, and overall success rate. Access site complications, procedure time, contrast volume needed, and ambulation time post-procedure were likewise evaluated. The definition of MACE was reviewed in each study, and was determined as the composite of myocardial infarction, death, and target vessel revascularization. The pooled data were analyzed with

*Corresponding author: Waleed E Ali, Department of Internal Medicine, Cape Fear Valley Medical Center, 1638 Owen Drive, Fayetteville, North Carolina 28304, USA, Tel: 9106351334; E-mail: waleeed197897@yahoo.com

Received April 01, 2018; Accepted May 02, 2018; Published May 07, 2018

Citation: Ali WE, Bahekar AA, Ejah SU (2018) Meta-analysis Comparing the Transradial and Transfemoral Approaches for Percutaneous Coronary Intervention in Elderly Patients. J Cardiovasc Dis Diagn 6: 322. doi: 10.4172/2329-9517.1000322

Copyright: ( 2018 Ali WE, et al. This is an open-access article distributed under the terms of the Creative Commons Attribution License, which permits unrestricted use, distribution, and reproduction in any medium, provided the original author and source are credited. 
Review Manager Version 5.3 (RevMan software, available from http:// tech.cochrane.org/revman. We used the Mantel-Haenszel statistical method with random effects. Continuous variables were analyzed using mean and standard deviation. Effect size for individual clinical outcomes was estimated using odds ratios (ORs) and confidence intervals (CIs). The heterogeneity across studies was examined by using the $\mathrm{Q}$ test and quantified with the $\mathrm{I} 2$ test. A value of $>50 \%$ and a $\mathrm{P}$ value of $<0.1$ were considered significant.

\section{Results}

A total of 4,066 patients were included in our study, with 2,540 in TR group versus 1,526 in TF group. We identified 2 randomized controlled trials and 11 observational studies. The randomized controlled trails included the OCTOPLUS trial (Comparison of Transradial and Transfemoral Approaches for Coronary Angiography and Angioplasty in Octogenarians) and the trial by Achenbach et al. (Transradial Versus Transfemoral Approach for Coronary
Angiography and Intervention in Patients Above 75 Years of Age). The baseline characteristics of all included studies are summarized in Tables 1 and 2. One randomized trial had a Jadad Score of 2 for lack of blinding (Table 3). The observational studies were considered highquality based on the Newcastle-Ottawa Scale (NOS) form (Table 4). There were no significant differences between the 2 groups in MACE (OR 0.64, 95\% CI 0.40-1.02, $\mathrm{P}=0.06$ ), cerebrovascular accidents (OR 1.82, 95\% CI $0.28-11.68, \mathrm{P}=0.53$ ), revascularization (OR $1.14,95 \%$ CI 0.43-3.04, $\mathrm{P}=0.79$ ), pseudoaneurysm formation (OR 0.33, 95\% CI 0.08-1.32, $\mathrm{P}=0.12$ ), volume of contrast given (mean difference [MD] $-1.11,95 \%$ CI $-19.57-17.35, \mathrm{P}=0.91$ ), and procedure time (MD 0.40, 95\% CI -3.62-4.42, $\mathrm{P}=0.84$ ) (Figures 2-6). However, Major bleeding and ambulation time were lower in the TR group (OR 0.49,95\% CI $0.33-0.71, \mathrm{P}=0.0002$ and $\mathrm{MD}-10.51,95 \% \mathrm{CI}-19.33--1.69, \mathrm{P}=0.02$, respectively) (Figures 7 and 8 ). On the other hand, the success rate remained higher in TF group (OR 1.71, 95\% CI 1.09-2.67, $\mathrm{P}=0.02$ ) (Figure 9).

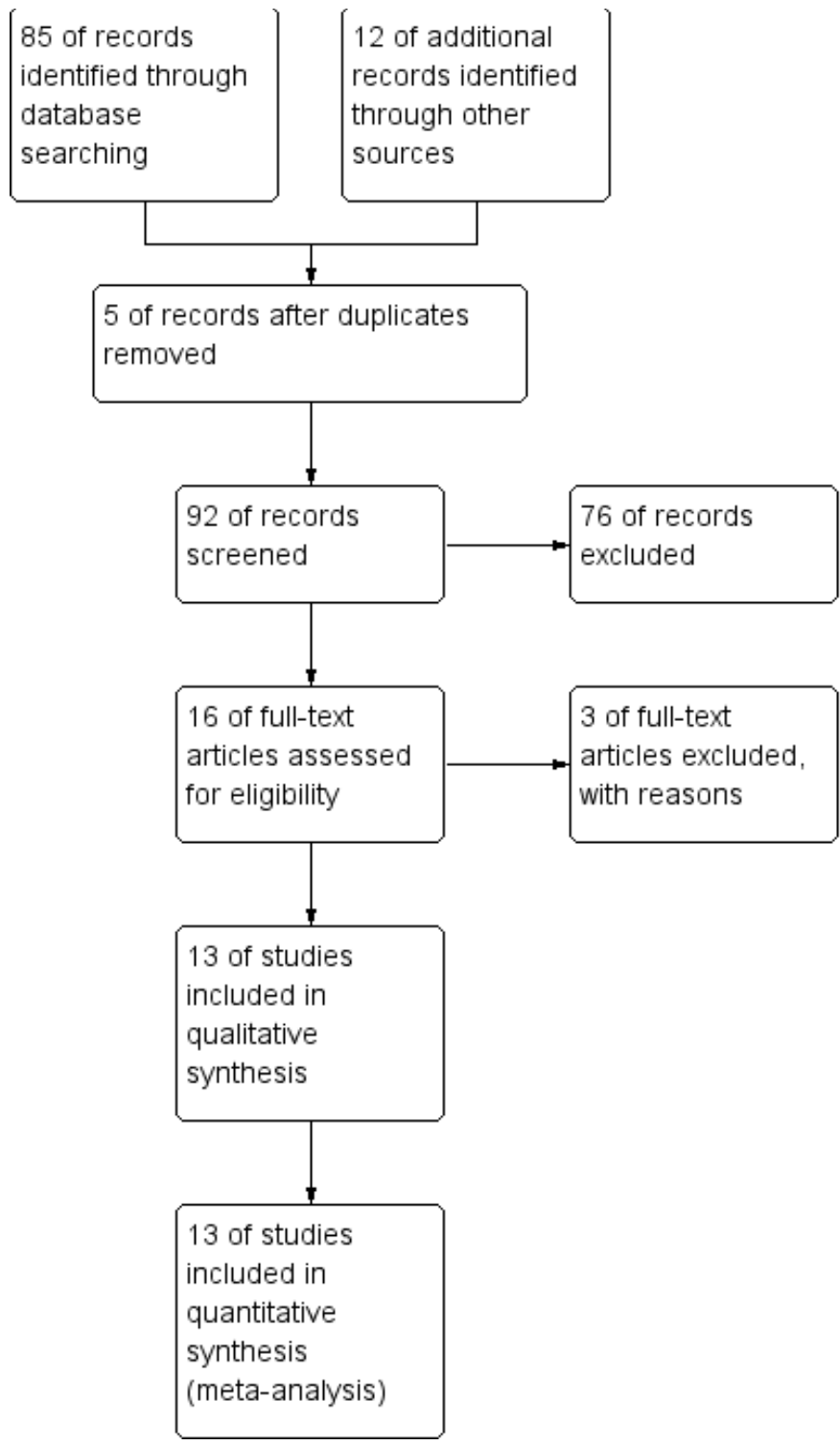

Figure 1: The study flow diagram. 
Citation: Ali WE, Bahekar AA, Ejah SU (2018) Meta-analysis Comparing the Transradial and Transfemoral Approaches for Percutaneous Coronary Intervention in Elderly Patients. J Cardiovasc Dis Diagn 6: 322. doi: 10.4172/2329-9517.1000322

Page 3 of 8

\begin{tabular}{|c|c|c|c|c|c|c|c|c|}
\hline Study & Year & Age & Type of study & $\begin{array}{l}\text { Duration of } \\
\text { study }\end{array}$ & Study outcomes & $\mathrm{PCl}$ type & $\begin{array}{c}\text { cross-over (TR } \\
\text { to TF) }\end{array}$ & $\begin{array}{l}\text { TR operator } \\
\text { experience }\end{array}$ \\
\hline $\begin{array}{l}\text { Achenbach et al. } \\
\text { [20] }\end{array}$ & 2008 & $>75$ & Randomized trial & 17 months & $\begin{array}{c}\text {-Fluoroscopic time } \\
\text {-contrast volume } \\
\text {-Number of catheters used } \\
\text {-Pseudoaneurysm/ Major events }\end{array}$ & $\begin{array}{l}\text { Elective/ } \\
\text { ACS }\end{array}$ & $\begin{array}{l}9 \% \text { crossed } \\
\text { over to TF }\end{array}$ & Experienced \\
\hline Bertrand et al. [21] & 2010 & $>80$ & Observation & $2002-2008$ & $\begin{array}{l}\text {-Mortality/MACE } \\
\text {-Revascularization } \\
\text { CVA/MI/Bleeding }\end{array}$ & $\begin{array}{l}\text { Elective/MI } \\
\mathrm{PCl} \text { of LM }\end{array}$ & Not reported & NA \\
\hline Jimenez et al. [22] & 2011 & $>75$ & Observation & 4 years & $\begin{array}{c}\text {-Success rate } \\
\text {-Fluoroscopic time / Volume of contrast } \\
-30 \text { day mortality } \\
-1 \text { year mortality }\end{array}$ & STEMI & Not reported & NA \\
\hline Hu et al. [23] & 2012 & $>80$ & Observation & 2003-2007 & $\begin{array}{l}\text {-Revascularization/ Success rate } \\
\text {-Mortality/Volume of contrast } \\
\text {-MI/Vascular complications } \\
\text {-Ambulation time/Bleeding }\end{array}$ & ACS & $\begin{array}{l}9.8 \% \text { crossed } \\
\text { over to TF }\end{array}$ & Experienced \\
\hline Jaffe et al. [24] & 2007 & $>80$ & Observation & 2000-2004 & $\begin{array}{c}\text {-Success rate } \\
\text {-Ambulation time } \\
\text {-Vascular complication } \\
\text {-Revascularization/CVA }\end{array}$ & Elective/ACS & $\begin{array}{l}11 \% \text { crossed } \\
\text { over to TF }\end{array}$ & Experienced \\
\hline Jin et al. [25] & 2017 & $>65$ & Observation & $\begin{array}{l}\text { One year } \\
(2010)\end{array}$ & $\begin{array}{l}\text {--Hospital cost/ LOS } \\
\text {-MACE } \\
\text {-Bleeding }\end{array}$ & Elective/ACS & No cross- over & NA \\
\hline Klinke et al. [26] & 2004 & $\geq 80$ & Observation & $1998-2000$ & $\begin{array}{c}\text {-Success rate/Procedure time } \\
\text {-Mortality/ LOS }\end{array}$ & Elective/ACS & Not reported & Experienced \\
\hline Koutouzis et al. [27] & 2010 & $\geq 80$ & Observation & $2002-2007$ & $\begin{array}{c}\text {-MACE/Mortality/CVA } \\
\text {-Vascular complication } \\
\text {-Revascularization /Contrast volume } \\
\text {-Success rate/Time }\end{array}$ & Elective/ACS & $\begin{array}{l}\text { 18.3\% Crossed } \\
\text { over to TF / } \\
\text { Trans- brachial }\end{array}$ & Experienced \\
\hline Louvard et al. [28] & 2004 & $>80$ & Randomized & $2001-2003$ & $\begin{array}{c}\text {-Bleeding } \\
\text {-Vascular complication } \\
\text {-CVA }\end{array}$ & Elective/ACS & $\begin{array}{l}1 \% \text { crossed } \\
\text { over to TF }\end{array}$ & Experienced \\
\hline Secco et al. [29] & 2013 & $>75$ & Observation & 2008-2011 & $\begin{array}{l}\text { Bleeding } \\
\text {-Door to balloon time } \\
\text {-Time of arterial puncture } \\
\text {-Time of balloon inflation }\end{array}$ & STEMI & Not reported & NA \\
\hline Tammam et al. [30] & 2017 & $\geq 80$ & Observation & 2010-2014 & $\begin{array}{c}\text {-Success rate/ Contrast volume } \\
\text {-Bleeding } \\
\text {-Mortality } \\
\text {-LOS }\end{array}$ & Elective/ACS & Not reported & NA \\
\hline Yan et al. [31] & 2008 & $\geq 65$ & Observation & 2005-2007 & $\begin{array}{c}\text {-Success rate/ Procedure time } \\
\text {-Bleeding } \\
\text {-vascular complication } \\
\text { - MACE/Revascularization }\end{array}$ & ACS & Not reported & Experienced \\
\hline Ziakas et al. [32] & 2007 & $\geq 70$ & Observation & $2000-2005$ & $\begin{array}{l}\text { MACE/Revascularization } \\
\text { Procedure success/procedure time }\end{array}$ & ACS & $\begin{array}{l}8 \% \text { crossed } \\
\text { over to TF }\end{array}$ & Experienced \\
\hline
\end{tabular}

Table 1: Characteristics of included studies.

\begin{tabular}{|c|c|c|c|c|c|c|c|c|c|c|}
\hline Study & Year & Patients & Age \pm SD & Males & DM & HTN & Prior PCI & Prior CABG & Stable CAD & ACS \\
\hline \multirow{2}{*}{ Achenbach et al. [20] } & \multirow{2}{*}{2008} & TR152 & TR $78 \pm 3$ & TR 70 & \multirow{2}{*}{ NA } & \multirow{2}{*}{ NA } & \multirow{2}{*}{ NA } & TR 12 & \multirow{2}{*}{ NA } & \multirow{2}{*}{ NA } \\
\hline & & TF 155 & TF $78 \pm 3$ & TF 68 & & & & TF 19 & & \\
\hline \multirow{2}{*}{ Bertrand et al. [21] } & \multirow{2}{*}{2010} & TR 90 & TR $85 \pm 3$ & TR 51 & TR 22 & TR 69 & TR 13 & TR 2 & TR 7 & TR 81 \\
\hline & & TF 13 & TF $82 \pm 3$ & TF 5 & TF 5 & TF 8 & TF 2 & TF 0 & TF 1 & TF 11 \\
\hline \multirow{2}{*}{ Jimenez et al. [22] } & \multirow{2}{*}{2011} & TR 273 & $\begin{array}{c}\text { TR } 79.9 \pm \\
3.75\end{array}$ & TR 150 & TR 63 & TR 130 & TR 51 & TR 3 & TR 0 & TR 273 \\
\hline & & TF 83 & $\begin{array}{c}\text { TF } 81.48 \pm \\
4.58\end{array}$ & TF 41 & TF 22 & TF 42 & TF 14 & TF 0 & TF 0 & TF 83 \\
\hline \multirow{2}{*}{ Hu et al. [23] } & \multirow{2}{*}{2012} & TR 112 & $\begin{array}{c}\text { TR } 82.68 \pm \\
2.63\end{array}$ & TR 76 & TR 44 & TR 84 & TR 12 & TR 5 & TR 0 & TR 112 \\
\hline & & TF 156 & $\begin{array}{c}\text { TF } 82.75 \pm \\
3.31\end{array}$ & TF 96 & TF 57 & TF 106 & TF 16 & TF 8 & TF 0 & TF 156 \\
\hline \multirow{2}{*}{ Jaffe et al. [24] } & \multirow{2}{*}{2006} & TR 97 & TR $82 \pm 2$ & TR 65 & TR 20 & TR 83 & TR 11 & TR 4 & TR 71 & TR 26 \\
\hline & & TF 131 & TF $83 \pm 4$ & TF 70 & TF 24 & TF 108 & TF 21 & TF 10 & TF 102 & TF 29 \\
\hline
\end{tabular}


Citation: Ali WE, Bahekar AA, Ejah SU (2018) Meta-analysis Comparing the Transradial and Transfemoral Approaches for Percutaneous Coronary Intervention in Elderly Patients. J Cardiovasc Dis Diagn 6: 322. doi: 10.4172/2329-9517.1000322

Page 4 of 8

\begin{tabular}{|c|c|c|c|c|c|c|c|c|c|c|}
\hline \multirow{2}{*}{ Jin et al. [25] } & \multirow{2}{*}{2017} & TR 1033 & TR $71 \pm 8.4$ & TR 672 & TR 278 & TR 711 & TR 179 & TR 12 & TR 274 & TR 730 \\
\hline & & TF 96 & TF $71.7 \pm 3.9$ & TF 48 & TF 72 & TF 141 & TF 56 & TF 19 & TF 56 & TF 133 \\
\hline \multirow[t]{2}{*}{ Klinke et al. [26] } & \multirow[t]{2}{*}{2004} & TR 125 & $\begin{array}{c}\text { TR } 83.3 \pm \\
2.5\end{array}$ & TR 75 & TR 19 & TR 83 & TR 26 & TR 22 & TR 54 & TR 71 \\
\hline & & TF 128 & TF $83.4 \pm 2.5$ & TF 56 & TF 38 & TF 98 & TF 38 & TF 23 & TF 65 & TF 63 \\
\hline \multirow{2}{*}{ Koutouzis et al. [27] } & \multirow{2}{*}{2010} & TR 40 & TR $84 \pm 2.7$ & TR 15 & TR 3 & TR 18 & TR 4 & \multirow{2}{*}{ NA } & \multirow{2}{*}{ NA } & \multirow{2}{*}{ NA } \\
\hline & & TF 301 & TF $84 \pm 2.9$ & TF 171 & TF 39 & TF 152 & TF 35 & & & \\
\hline \multirow[t]{2}{*}{ Louvard et al. [28] } & \multirow[t]{2}{*}{2004} & TR 192 & $\begin{array}{c}\text { TR } 82.6 \pm \\
2.7\end{array}$ & TR 105 & TR 17 & TR 111 & TR 28 & TR 15 & TR 86 & TR 106 \\
\hline & & TF 185 & TF $83 \pm 3.1$ & TF 92 & TF 34 & TF 120 & TF 45 & TF 20 & TF 74 & TF 111 \\
\hline \multirow{2}{*}{ Secco et al. [29] } & \multirow{2}{*}{2013} & TR 177 & TR $81.6 \pm 4$ & TR 101 & TR 36 & TR 121 & TR 14 & TR 6 & \multirow{2}{*}{ NA } & TR 177 \\
\hline & & TF 106 & TF $83.3 \pm 4$ & TF 45 & TF 19 & TF 60 & TF 11 & TF 3 & & TF 106 \\
\hline \multirow{2}{*}{ Tammam et al. [30] } & \multirow{2}{*}{2017} & TR 218 & TR $83 \pm 3$ & TR 145 & TR 78 & TR 174 & TR 71 & \multirow{2}{*}{ NA } & TR 132 & TR 86 \\
\hline & & TF 73 & TF $84 \pm 4$ & TF 52 & TF 39 & TF 57 & TF 35 & & TF 40 & TF 33 \\
\hline \multirow[t]{2}{*}{ Yan et al. [31] } & \multirow[t]{2}{*}{2008} & TR 57 & $\begin{array}{c}\text { TR } 70.3 \pm \\
7.5\end{array}$ & TR 43 & TR 13 & TR 24 & \multirow[t]{2}{*}{ NA } & \multirow[t]{2}{*}{ NA } & TR 0 & TR 57 \\
\hline & & TF 46 & TF $71.4 \pm 8.4$ & TF 34 & TF 11 & TF 22 & & & TF 0 & TF 46 \\
\hline \multirow[t]{2}{*}{ Ziakas et al. [32] } & \multirow[t]{2}{*}{2007} & TR 87 & $\begin{array}{c}\text { TR } 75.7 \pm \\
4.5\end{array}$ & TR 56 & TR 18 & TR 32 & TR 12 & TR 2 & \multirow[t]{2}{*}{ NA } & TR 87 \\
\hline & & TF 68 & TF $77.7 \pm 4.9$ & TF 43 & TF 9 & TF 24 & TF18 & TF 11 & & TF 68 \\
\hline
\end{tabular}

SD: Standard Deviation; DM: Diabetes; HTN: Hypertension; PCI: Percutaneous Coronary Intervention; CABG: Coronary Artery Bypass Graft; CAD: Coronary Artery Disease; ACS: Acute Coronary Syndrome; TR: Transradial; TF: Transfemoral; NA: Not Available or Difficult to Extract.

Table 2: Patients baseline characteristics in each study.

\begin{tabular}{|c|c|c|}
\hline Rating Scale List & Achenbach et al. [20] & Louvard et al. [28] \\
\hline Was the study described as random & Yes & Yes \\
\hline Was the randomization described and appropriate & Yes & Yes \\
\hline Was the study described as double- blind & No & Yes \\
\hline Was the method of double blinding appropriate & Yes & Yes \\
\hline Was there a description of dropouts and withdrawals & 2 & Yes \\
\hline Jadad Score & 5 & \\
\hline
\end{tabular}

Table 3: Randomized studies quality assessment using Oxford Quality Scoring System. Jadad score $\geq 3$ considered high quality.

\begin{tabular}{|c|c|c|c|c|c|c|c|c|c|}
\hline \multirow[b]{2}{*}{ Study } & \multicolumn{4}{|c|}{ Selection } & \multirow[b]{2}{*}{$\begin{array}{c}\text { Comparability of } \\
\text { the cohort }\end{array}$} & \multicolumn{3}{|c|}{ Outcome } & \multirow[b]{2}{*}{ Total score } \\
\hline & $\begin{array}{c}\text { Representativeness } \\
\text { of the exposed } \\
\text { cohort }\end{array}$ & $\begin{array}{c}\text { Selection } \\
\text { of the non- } \\
\text { exposed } \\
\text { cohort }\end{array}$ & $\begin{array}{l}\text { Ascertainment } \\
\text { of exposure }\end{array}$ & $\begin{array}{c}\text { Outcome not } \\
\text { present at } \\
\text { baseline }\end{array}$ & & $\begin{array}{c}\text { Assessment } \\
\text { of outcome }\end{array}$ & $\begin{array}{c}\text { Enough follow-up } \\
\text { duration }\end{array}$ & $\begin{array}{l}\text { Adequate } \\
\text { follow-up }\end{array}$ & \\
\hline Bertrand et al. [20] & * & * & * & * & $* *$ & * & * & * & 9 \\
\hline Jimenez et al. [22] & * & * & * & * & ** & * & * & * & 9 \\
\hline Hu et al. [23] & * & * & * & * & * & * & * & * & 8 \\
\hline Jaffe et al. [24] & * & * & * & * & $* *$ & * & * & * & 8 \\
\hline Jin et al. [25] & * & * & * & * & $* *$ & * & * & * & 9 \\
\hline Klinke et al. [26] & * & * & * & * & ** & * & * & * & 9 \\
\hline $\begin{array}{l}\text { Koutouzis et al. } \\
\text { [27] }\end{array}$ & * & * & * & * & * & * & * & * & 8 \\
\hline Secco et al. [29] & * & * & * & * & $* *$ & * & * & * & 9 \\
\hline Tammam et al. [30] & * & * & * & * & ** & * & * & * & 9 \\
\hline Yan et al. [31] & * & * & * & * & ** & * & * & * & 9 \\
\hline Ziakas et al. [32] & * & * & * & * & $* *$ & * & * & * & 9 \\
\hline
\end{tabular}

Table 4: Newcastle-Ottawa scale (NOS) for assessing quality of observational studies. The scale assigns four points for selection, two points for comparability and three points for outcome. Score of 5-6 considered as moderate quality and 7-9 as high quality.

\section{Discussion}

The transradial route in PCI has gained increased popularity among interventional cardiologists for younger patients. The approach is widely preferred in Europe and Asia, while the similar trend is lagging behind in the United States. Previous studies in unselected patients undergoing the TR approach demonstrated non-inferior outcomes compared to the TF route, with even better results in terms of bleeding and vascular access complications [9-11]. Limited studies with small sample sizes were available for the same comparison in the elderly population. We therefore conducted this meta-analysis of 13 studies, including 2 randomized trials and 11 up-to-date observational studies (Table 2). Eleven studies recruited patients $\geq 70$ years old, while 2 studies recruited patients $\geq 65$ years old (Table 1). Our results showed that the TR approach was comparable to the TF route in elderly patients, and achieved a similar safety profile, with similar rates of MACE, cerebrovascular accidents, and revascularization outcomes (Figures 1-3). Additionally, no difference was found between the 2 groups regarding the procedure time, volume of contrast needed, and 
Citation: Ali WE, Bahekar AA, Ejah SU (2018) Meta-analysis Comparing the Transradial and Transfemoral Approaches for Percutaneous Coronary Intervention in Elderly Patients. J Cardiovasc Dis Diagn 6: 322. doi: 10.4172/2329-9517.1000322

\begin{tabular}{|c|c|c|c|c|c|c|c|c|c|c|}
\hline Study or Subgroup & $\begin{array}{r}\text { TR } \\
\text { Events }\end{array}$ & & $\begin{array}{r}\mathrm{TF} \\
\text { Events }\end{array}$ & Total & Weight & $\begin{array}{l}\text { Odds Ratio } \\
\text { M-H, Random, } 95 \% \mathrm{Cl}\end{array}$ & & $\begin{array}{r}\text { Odds } \mathrm{F} \\
\text { M-H, Rando }\end{array}$ & $\begin{array}{l}\text { Ratio } \\
\mathrm{m}, 95 \% \mathrm{Cl}\end{array}$ & \\
\hline Bertrand et al 2010 & 17 & 90 & 4 & 13 & $10.0 \%$ & $0.52[0.14,1.90]$ & & $\because$ & E & \\
\hline Hu et al 2012 & 12 & 112 & 15 & 156 & $18.9 \%$ & $1.13[0.51,2.51]$ & & 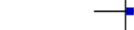 & & \\
\hline Jaffe et al 2007 & 5 & 97 & 26 & 131 & $14.5 \%$ & $0.22[0.08,0.59]$ & & - & & \\
\hline Jin et al 2017 & 30 & 1033 & 11 & 196 & $21.5 \%$ & $0.50[0.25,1.02]$ & & - & & \\
\hline Koutouzis et al 2009 & 2 & 40 & 21 & 301 & $8.0 \%$ & $0.70[0.16,3.11]$ & & & & \\
\hline Louvard et al 2004 & 8 & 89 & 5 & 97 & $11.8 \%$ & $1.82[0.57,5.78]$ & & & $\vec{*}$ & \\
\hline Yan 2008 & 3 & 87 & 4 & 68 & $7.6 \%$ & $0.57[0.12,2.64]$ & & & & \\
\hline Ziakas et al 2007 & 3 & 87 & 4 & 68 & $7.6 \%$ & $0.57[0.12,2.64]$ & & & & \\
\hline Total $(95 \% \mathrm{Cl})$ & & 1635 & & 1030 & $100.0 \%$ & $0.64[0.40,1.02]$ & & & & \\
\hline Total events & 80 & & 90 & & & & & & & \\
\hline $\begin{array}{l}\text { Heterogeneity: Tau }= \\
\text { Test for overall effect: }\end{array}$ & $\begin{array}{l}.13 ; \mathrm{Chi}^{2} \\
=1.90(\mathrm{P}\end{array}$ & $\begin{array}{l}=10.0 \\
=0.08\end{array}$ & $8, d f=7$ & $P=0.1$ & $8) i^{2}=319$ & & 0.01 & 0.1 & 1 & 100 \\
\hline
\end{tabular}

Figure 2: Forest plot of major cardiovascular and cerebrovascular events (MACE) outcome in transradial (TR) versus transfemoral (TF) approach in percutaneous coronary intervention (PCI).

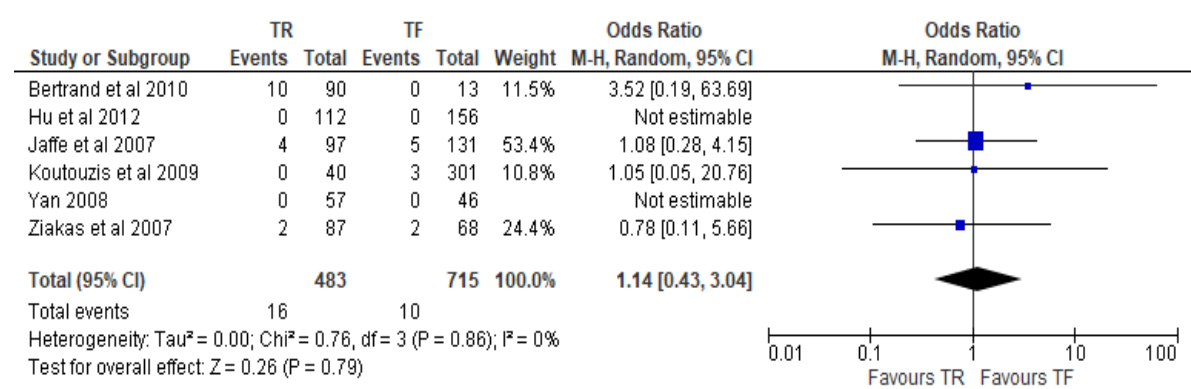

Figure 3: Forest plot of revascularization outcome in transradial (TR) versus transfemoral (TF) approach in percutaneous coronary intervention $(\mathrm{PCl})$.

\begin{tabular}{|c|c|c|c|c|c|c|c|c|c|c|}
\hline Study or Subgroup & $\begin{array}{r}\text { TR } \\
\text { Events } \\
\end{array}$ & Total & $\begin{array}{r}\text { TF } \\
\text { Events }\end{array}$ & Total & Weight & $\begin{array}{c}\text { Odds Ratio } \\
\text { M-H, Random, } 95 \% \mathrm{Cl}\end{array}$ & \multicolumn{4}{|c|}{$\begin{array}{c}\text { Odds Ratio } \\
\text { M- } \mathrm{H}, \text { Random, } 95 \% \mathrm{Cl}\end{array}$} \\
\hline Bertrand et al 2010 & 1 & 90 & 0 & 13 & $32.8 \%$ & $0.45[0.02,11.69]$ & & - & & \\
\hline Jaffe et al 2007 & 1 & 97 & 0 & 131 & $33.6 \%$ & $4.09[0.16,101.44]$ & & & & \\
\hline Koutouzis et al 2009 & 0 & 40 & 0 & 301 & & Not estimable & & & & \\
\hline Louvard et al 2004 & 1 & 185 & 0 & 192 & $33.7 \%$ & $3.13[0.13,77.33]$ & & & & \\
\hline Ziakas et al $200 ?$ & 0 & 87 & 0 & 68 & & Not estimable & & & & \\
\hline Total $(95 \%$ Cl) & & 499 & & 705 & $100.0 \%$ & $1.82[0.28,11.68]$ & & & & \\
\hline Total events & 3 & & 0 & & & & & & & \\
\hline $\begin{array}{l}\text { Heterogeneity: Tau² } \\
\text { Test for overall effect: }\end{array}$ & $\begin{array}{l}.00 ; \mathrm{Chi}^{2} \\
=0.63(\mathrm{P}\end{array}$ & $\begin{array}{l}=1.08, \\
=0.53\end{array}$ & $d f=2(P$ & $=0.58$ & 3); $1^{2}=0 \%$ & & 0.01 & $\begin{array}{l}1 \\
\text { Favours TR }\end{array}$ & 1 Favours TF & 100 \\
\hline
\end{tabular}

Figure 4: Forest plot of cerebrovascular accidents outcome in transradial (TR) versus transfemoral (TF) approach in percutaneous coronary intervention $(\mathrm{PCl})$.

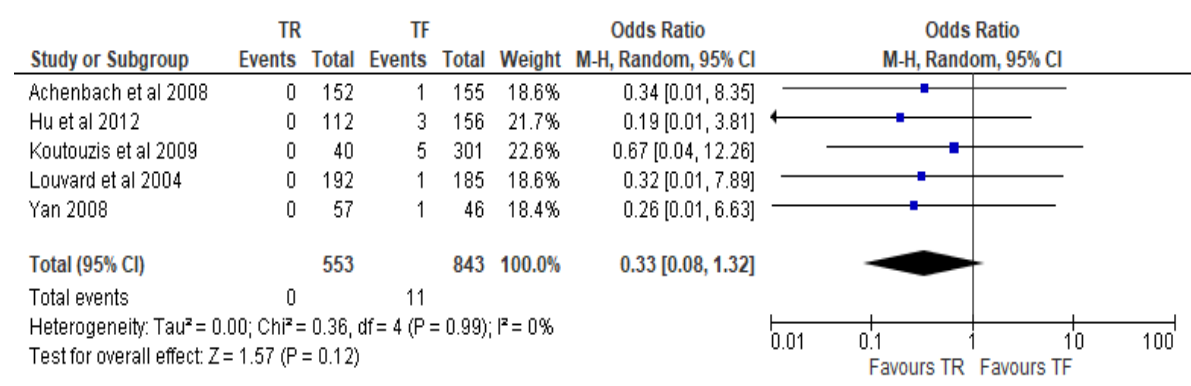

Figure 5: Forest plot of pseudoaneurysm outcome in transradial (TR) versus transfemoral (TF) approach in percutaneous coronary intervention $(\mathrm{PCl})$. 
Citation: Ali WE, Bahekar AA, Ejah SU (2018) Meta-analysis Comparing the Transradial and Transfemoral Approaches for Percutaneous Coronary Intervention in Elderly Patients. J Cardiovasc Dis Diagn 6: 322. doi: 10.4172/2329-9517.1000322

\begin{tabular}{|c|c|c|c|c|c|c|c|c|c|c|c|c|}
\hline \multirow[b]{2}{*}{ Study or Subgroup } & \multicolumn{3}{|c|}{ TR } & \multicolumn{3}{|c|}{ TF } & \multicolumn{3}{|c|}{ Mean Difference } & \multirow{2}{*}{\multicolumn{2}{|c|}{$\begin{array}{c}\text { Mean Difference } \\
\text { IV, Random, } 95 \% \mathrm{Cl}\end{array}$}} & \\
\hline & Mean & SD & Total & Mean & SD & Total & Weight & IV, Random, $95 \% \mathrm{Cl}$ & & & & \\
\hline Achenbach et al 2008 & 88 & 33 & 152 & 79 & 28 & 155 & $15.2 \%$ & $9.00[2.15,15.85]$ & & & - & \\
\hline Bertrand et al 2010 & 270 & 114 & 90 & 263 & 91 & 13 & $6.6 \%$ & $7.00[-47.79,61.79]$ & & & & \\
\hline Hu et al 2012 & 227 & 48 & 112 & 205 & 39 & 156 & $14.7 \%$ & $22.00[11.21,32.79]$ & & & $\rightarrow$ & \\
\hline Jaffe et al 2007 & 224 & 46 & 97 & 182 & 20 & 131 & $14.9 \%$ & $42.00[32.23,51.77]$ & & & $\rightarrow$ & \\
\hline Jimenez et al 2011 & 218 & 95 & 273 & 230 & 94 & 83 & $12.5 \%$ & $-12.00[-35.15,11.15]$ & & & & \\
\hline Koutouzis et al 2009 & 164 & 55 & 40 & 193 & 97 & 301 & $13.1 \%$ & $-29.00[-49.26,-8.74]$ & & & & \\
\hline Tammam et al 2017 & 145 & 73 & 218 & 180 & 86 & 73 & $12.7 \%$ & $-35.00[-56.98,-13.02]$ & & & & \\
\hline Ziakas et al 2007 & 212 & 92 & 87 & 238 & 114 & 68 & $10.3 \%$ & $-26.00[-59.29,7.29]$ & & & & \\
\hline Total $(95 \% \mathrm{Cl})$ & & & 1069 & & & 980 & $100.0 \%$ & $-1.11[-19.57,17.35]$ & & & & \\
\hline $\begin{array}{l}\text { Heterogeneity: } \operatorname{Tau}^{2}=5 \\
\text { Test for overall effect: } Z\end{array}$ & $\begin{aligned} & 1.13 ; \mathrm{Ch} \\
= & 0.12(\mathrm{P}\end{aligned}$ & $=0.91$ & & & & 1001); $\left.\right|^{2}$ & $z=92 \%$ & & & $\begin{array}{l}-50 \\
\text { Favours TR }\end{array}$ & 2 Favours TF & $100^{\circ}$ \\
\hline
\end{tabular}

Figure 6: Forest plot of volume of contrast administered in transradial (TR) versus transfemoral (TF) approach in percutaneous coronary intervention $(\mathrm{PCl})$

\begin{tabular}{|c|c|c|c|c|c|c|c|c|c|c|}
\hline Study or Subgroup & & & Weight & Odds Ratio & & \multicolumn{2}{|c|}{$\begin{array}{c}\text { Odds Ratio } \\
\text { M-H, Random, } 95 \% \mathrm{Cl}\end{array}$} & \\
\hline Achenbach et al 2008 & 0 & 36 & 3 & 43 & $1.6 \%$ & $0.16[0.01,3.17]$ & & & & \\
\hline Bertrand et al 2010 & 13 & 90 & 3 & 13 & $7.0 \%$ & $0.56[0.14,2.32]$ & & & - & \\
\hline Hu et al 2012 & 3 & 112 & 15 & 156 & $8.7 \%$ & $0.26[0.07,0.92]$ & & & & \\
\hline Jaffe et al 2007 & 4 & 97 & 19 & 131 & $11.2 \%$ & $0.25[0.08,0.77]$ & & & & \\
\hline Jin et al 2017 & 92 & 1033 & 23 & 196 & $51.2 \%$ & $0.74[0.45,1.19]$ & & $\rightarrow$ & & \\
\hline Koutouzis et al 2009 & 0 & 40 & 5 & 301 & $1.7 \%$ & $0.67[0.04,12.26]$ & & & & \\
\hline Louvard et al 2004 & 0 & 192 & 8 & 185 & $1.7 \%$ & $0.05[0.00,0.95]$ & & & & \\
\hline Secco et al 2012 & 2 & 177 & 5 & 106 & $5.1 \%$ & $0.23[0.04,1.21]$ & & & & \\
\hline Tammam et al 2017 & 8 & 218 & 5 & 73 & $10.5 \%$ & $0.52[0.16,1.64]$ & & $=$ & - & \\
\hline Yan 2008 & 0 & 57 & 1 & 46 & $1.4 \%$ & $0.26[0.01,6.63]$ & & & & \\
\hline Total $(95 \% \mathrm{Cl})$ & & 2052 & & 1250 & $100.0 \%$ & $0.49[0.33,0.71]$ & & & & \\
\hline Total events & 122 & & 87 & & & & & & & \\
\hline $\begin{array}{l}\text { Heterogeneity: } \operatorname{Tau}^{2}= \\
\text { Test for overall effect: }\end{array}$ & $\begin{array}{l}01 ; \mathrm{Chi}^{2}= \\
=3.72(\mathrm{P}\end{array}$ & $\begin{array}{l}=9.23 \\
=0.00\end{array}$ & $\begin{array}{l}d f=9(P= \\
b 2)\end{array}$ & $0.42)$; & $z^{2}=2 \%$ & & 0.01 & $\begin{array}{l}0.1 \\
\text { Favours TR }\end{array}$ & $\begin{array}{l}1 \\
\text { Favours TF }\end{array}$ & 100 \\
\hline
\end{tabular}

Figure 7: Forest plot of major bleeding outcome in transradial (TR) versus transfemoral (TF) approach in percutaneous coronary intervention (PCl).

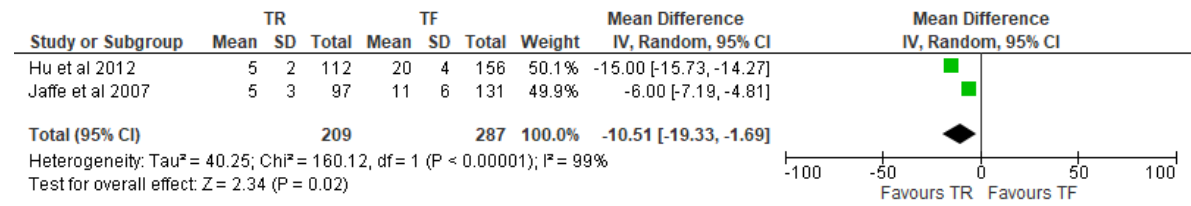

Figure 8: Forest plot of post procedure ambulation time in transradial (TR) versus transfemoral (TF) approach in percutaneous coronary intervention $(\mathrm{PCl})$

\begin{tabular}{|c|c|c|c|c|c|c|c|c|c|c|}
\hline Study or Subgroup & & & \multicolumn{3}{|c|}{ Odds Ratio } & \multicolumn{2}{|c|}{$\begin{array}{c}\text { Odds Ratio } \\
\text { M-H, Random, } 95 \% \mathrm{Cl}\end{array}$} & \\
\hline Bertrand et al 2010 & 88 & 90 & 12 & 13 & $3.2 \%$ & $3.67[0.31,43.57]$ & & & & \\
\hline Hu et al 2012 & 108 & 112 & 149 & 156 & $12.7 \%$ & $1.27[0.36,4.44]$ & & & & \\
\hline Jaffe et al $200 ?$ & 91 & 97 & 124 & 131 & $15.8 \%$ & $0.86[0.28,2.63]$ & & & - & \\
\hline Klinke et al 2004 & 120 & 128 & 104 & 125 & $27.2 \%$ & $3.03[1.29,7.13]$ & & & - & \\
\hline Koutouzis et al 2009 & 38 & 40 & 280 & 301 & $9.0 \%$ & $1.43[0.32,6.32]$ & & & & \\
\hline Louvard et al 2004 & 86 & 89 & 93 & 97 & $8.5 \%$ & $1.23[0.27,5.67]$ & & & & \\
\hline Tammam et al 2017 & 209 & 218 & 69 & 73 & $13.6 \%$ & $1.35[0.40,4.51]$ & & & & \\
\hline Yan 2008 & 56 & 57 & 44 & 46 & $3.4 \%$ & $2.55[0.22,28.99]$ & & & & \\
\hline Ziakas et al 2007 & 85 & 87 & 64 & 68 & $6.7 \%$ & $2.66[0.47,14.95]$ & & & & \\
\hline Total $(95 \% \mathrm{Cl})$ & & 918 & & 1010 & $100.0 \%$ & $1.71[1.09,2.67]$ & & & & \\
\hline Total events & 881 & & 939 & & & & & & & \\
\hline $\begin{array}{l}\text { Heterogeneity: Tau }{ }^{2}= \\
\text { Test for overall effect: }\end{array}$ & $\begin{array}{l}0.00 ; \mathrm{Chi}^{-} \\
=2.35\end{array}$ & $\begin{array}{l}=4.49 \\
=0.0\end{array}$ & 2) $d f=8(P$ & $=0.81$ & 1); $\left.\right|^{2}=0 \%$ & & 0.01 & $\begin{array}{l}0.1 \\
\text { Favours TR }\end{array}$ & ${ }^{1}$ Favours TF & 100 \\
\hline
\end{tabular}

Figure 9: Forest plot of procedure success in transradial (TR) versus transfemoral (TF) approach in percutaneous coronary intervention (PCI).

arterial pseudoaneurysm formation (Figures 4-7). Moreover, the TR group had less bleeding risk (OR 0.49, 95\% CI 0.33-0.71, $\mathrm{P}=0.0002$ ) and earlier ambulation time (MD -10.51, 95\% CI -19.33- -1.69, $\mathrm{P}=0.02$ ) (Figures 4-10). These favorable outcomes remained even among elderly patients aged over 80 years [12-27].

Accessing the radial artery can be challenging in elderly patients with advanced peripheral vascular diseases and calcifications. Moreover, the relatively smaller diameter of the radial compared to the femoral artery and the risk of arterial spasm may limit the manipulation and size of the catheters used in PCI, which theoretically may result in prolonged procedure time and higher crossover rate to the femoral route. Interventional cardiologists are sometimes concerned about 


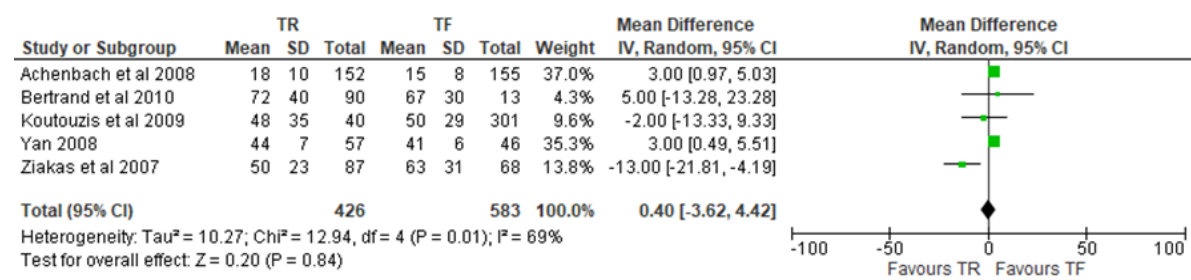

Figure 10: Forest plot of procedure time in transradial (TR) versus transfemoral (TF) approach in percutaneous coronary intervention (PCl).

longer procedure times and possible reperfusion delay in the acute setting. Studies on unselected patients had conflicting results, and some concluded that a longer procedure time was needed in the TR approach [11-13]. Bhat et al. [9] conducted a prospective randomized trial in an unselected age group, where 400 patients were allocated randomly to either the TR or TF approach for elective PCI. The procedure time was found to be longer in the TR group $(29 \pm 11.3$ min versus $27.3 \pm 12.4$ min, $\mathrm{P}$ value of 0.03$)$. However, in our analysis, the procedure time remained equal between the 2 modalities (MD 0.40, 95\% CI -3.624.42, $\mathrm{P}=0.84$ ) among elderly patients. On the other hand, Achenbach et al. [21] reported longer procedure times in TR patients $(18.1+10$ vs $15+8, \mathrm{P}=0.009)$ in randomized trial allocating 307 elderly patients to either procedure (TR vs TF). Recently, a large retrospective study was published where they recruited 1,229 patients over 65 years old who underwent PCI. Of the patients, $84 \%$ represented the TR group. There was no crossover from the TR to the TF approach. Although the procedure time was not reported for comparison, the success rate in achieving complete revascularization in the TR group, with no crossover, argued for potential benefit of the TR approach among elderly patients. Additionally, we noted that most of our included studies (Table 1) to assess the procedure time reported the experience of the radial operator, which is yet another factor for possible successful outcomes that needs to be considered in the TR approach. Although the result was reassuring, it remained heavily influenced by observational studies and had little input from randomized trials.

TR access site bleeding and hematoma was shown to be less compared to TF in previous studies conducted in unselected patients, with similar results as in those presenting with acute coronary syndrome (ACS) [14-16]. However, elderly patients inherit an increased risk of bleeding following PCI given their advanced age, presence of peripheral vascular disease, hypertension, and abnormal renal or liver function tests. This potential hemostatic benefit was assessed by only 1 randomized trial by Louvard et al. [28-32], where elderly patients over 80 years old were recruited and randomized to the TR or TF PCI approach for more than 2 years. The TR group exhibited a $3.7 \%$ rate of major bleeding, compared to $6.9 \%$ in the TF group. It is worthy to mention that glycoprotein IIb/IIIa inhibitors were not used in this study because of lack of government approval. We examined this outcome by enrolling studies defining major bleeding. Our criteria included post-PCI access site bleeding that required blood transfusion, resulted in a significant drop in hemoglobin, or led to the development of a hematoma necessitating longer hospital stay. Our result was in agreement with the randomized trial shown in Figure 4 and was consistent among all recruited studies (OR 0.49, 95\% CI 0.33-0.71, $\mathrm{P}=0.0002)$. Whether this reduction in major bleeding could translate into a mortality benefit among elderly patients remains unknown. We could not analyze the mortality benefit in our study due to lack of well-defined methods measuring the outcome in relation to bleeding risk between the observational studies. Nevertheless, the MATRIX (Minimizing Adverse Haemorrhagic Events by TRansradial Access
Site and Systemic Implementation of angioX) trial [17], a randomized study that allocated 8,404 patients to either TF or TR access, showed reduction in mortality in the TR group amongst unselected patients presenting with acute coronary syndrome (ACS). Interestingly, 25\% of their cohort was represented by an elderly population in their 80 s. Moreover, a similar observation was reported in previous studies [1819]. We believe further randomized trials are needed to provide robust evidence of a mortality benefit in the TR group based on low bleeding risk among elderly patients.

The criteria for procedural (PCI) success were not defined in most studies. It usually implies complete revascularization with Grade 3 Thrombolysis in Myocardial Infarction (TIMI) flow, and no procedural complications. Therefore, we analyzed all studies that presented their success rates in the clinical outcomes as a secondary end point. We found a higher success rate in favor of the TF group (OR 1.71, 95\% CI 1.09-2.67, $\mathrm{P}=0.02$ ) (Figure 8). However, a study by $\mathrm{Hu}$ et al. [23] reported similar success rates between the 2 groups, where success was defined as residual stenosis $<30 \%$, Grade 3 TIMI flow, and no procedural complications, as well as no peripheral vascular or cardiac events. Unfortunately, the analysis was not reliable because success was not precisely defined in majority of the studies. Nevertheless, it is reassuring that the rates of revascularization and MACE remained equal in our analysis (Figures 1 and 2), with no heterogeneity between the selected studies.

To assess the morbidity risk, we analyzed the volume of contrast given, which estimates the risk for kidney damage, and post-procedure ambulation time in the 2 groups. We found no difference in contrast volume given for each route (MD -1.11, 95\% CI -19.75-17.35, $\mathrm{P}=0.91$ ) (Figure 5), while patients who underwent the TR approach tended to ambulate earlier (MD -10.51, 95\% CI -19.33- -1.69, P=0.02) (Figure 10). Although, the later finding was evaluated by 2 studies with significant heterogeneity, almost all studies in unselected patients supported our observation [33-35]. Jin et al. [25] translated this advantage into earlier hospital discharge, shorter length of stay (1.9 days, 95\% CI 1.1-2.7 days), and less hospitalization costs overall.

We encountered some limitations in our study. First, most of the studies included in this analysis were observational studies with unavoidable selection bias. However, random-effect analysis model was applied in our analysis. Second, there was not enough data to compare the fluoroscopic time and cross-over between the 2 groups. Third, the precise definition of success rate was not provided in most studies, which made the analysis unreliable. Fourth, the level of experience of the radial operator could not be fully explored in observational studies; therefore, no subgroup analysis was performed.

\section{Conclusion}

In conclusion, the TR approach in elderly patients tended to have comparable outcomes to the TF route. Moreover, decreased risk of 
Citation: Ali WE, Bahekar AA, Ejah SU (2018) Meta-analysis Comparing the Transradial and Transfemoral Approaches for Percutaneous Coronary Intervention in Elderly Patients. J Cardiovasc Dis Diagn 6: 322. doi: 10.4172/2329-9517.1000322

bleeding and earlier ambulation time in the TR compared to the TF route may play in favor of early hospital discharges, and hence, less complications. A large randomized trial in this age group is needed to accurately assess TR procedural success and mortality compared to the TF approach.

\section{Acknowledgement}

None.

\section{Conflict of Interest}

None declared.

\section{Source of Funding}

None declared.

\section{References}

1. Yamashita Y, Shiomi H, Morimoto T, Yaku H, Kaji S, et al. (2017) Transradial versus transfemoral approach in patients undergoing primary percutaneous coronary intervention for ST-elevation acute myocardial infarction: insight from the CREDO-Kyoto AMI registry. Heart Vessels 32: 1448-1457.

2. Cortese B, Rigattieri S, Aranzulla TC, Russo F, Latib A, et al. (2018) Transradial versus transfemoral ancillary approach in complex structural, coronary, and peripheral interventions. Results from the multicenter ancillary registry: A study of the Italian Radial Club. Catheter Cardiovasc Interv 91: 97-102.

3. Choe JC, Cha KS, Choi JH, Kim BW, Park JS, et al. (2016) Comparison of frequency of bleeding and major adverse cardiac events after transradial versus transfemoral intervention in the recent antiplatelet era. Am J Cardiol 117: 1588-1595.

4. Shuvy M, Ko DT (2014) Bleeding after percutaneous coronary intervention: Can we still ignore the obvious? Open Heart 1: e000036.

5. Piper WD, Malenka DJ, Ryan TJ, Shubrooks SJ, O'Connor GT, et al. (2003) Predicting vascular complications in percutaneous coronary interventions. Am Heart J 145: 1022-1029.

6. Shanmugasundaram M (2011) Percutaneous coronary intervention in elderly patients; Is it beneficial? Tex Heart Inst J 38: 398403

7. Jadad AR, Moore RA, Carroll D (1996) Assessing the quality of reports of randomized clinical trials: is blinding necessary? Control Clin Trials 17: 1-12.

8. Stang A (2010) Critical evaluation of the Newcastle-Ottawa scale for the assessment of the quality of nonrandomized studies in meta-analyses. Eur $\mathrm{J}$ Epidemiol 25: 603-605.

9. Bhat FA, Changal KH, Raina H, Tramboo NA, Rather HA (2017) Transradial versus transfemoral approach for coronary angiography and angioplasty - A prospective, randomized comparison. BMC Cardiovasc Disord 17: 23.

10. Brueck M, Bandorski D, Kramer W, Wieczorek M, Holtgen R, et al. (2009) A randomized comparison of transradial versus transfemoral approach for coronary angiography and angioplasty. JACC Cardiovasc Interv 2: 1047-1054.

11. Tewari S, Sharma N, Kapoor A, Syal SK, Kumar S, et al. (2013) Comparison of transradial and transfemoral artery approach for percutaneous coronary angiography and angioplasty: A retrospective seven-year experience from a north Indian center. Indian Heart J 65: 378-387.

12. Schernthaner C, Hammerer M, Harb S, Heigert M, Hoellinger K, et al. (2017) Radial versus femoral access site for percutaneous coronary intervention in patients suffering acute myocardial infarction: A randomized prospective multicenter trial. Wien Klin Wochenschr 130: 182-189.

13. Dai Y, Li C, Zhang F, Yang J, Chang S, et al. (2018) Safety and efficacy of percutaneous coronary intervention via transradial versus transfemoral approach in bypass grafts. Angiology 69: 136-142.

14. Li H, Rha SW, Choi BG, Shim MS, Choi SY, et al. (2017) Transradial versus transfemoral intervention in ST-segment elevation myocardial infarction patients in Korean population. Korean J Intern Med 2016: 316.

15. Koifman E, Gaglia MA, Escarcega RO, Bernardo NL, Lager RA, et al. (2017) Comparison of transradial and transfemoral access in patients undergoing percutaneous coronary intervention for complex coronary lesions. Catheter Cardiovasc Interv 89: 640-646.
16. Wang $Y B, F u X H$, Wang $X C, G u X S$, Zhao $Y J$, et al. (2012) Randomized comparison of radial versus femoral approach for patients with STEM undergoing early $\mathrm{PCl}$ following intravenous thrombolysis. J Invasive Cardiol 24: $412-416$

17. Valgimigli M, Gagnor A, Calabro P, Frigoli E, Leonardi S, et al. (2015) Radia versus femoral access in patients with acute coronary syndromes undergoing invasive management: A randomized multicentre trial. Lancet 385: 2465-2476.

18. Jolly SS, Yusuf S, Cairns J, Niemela K, Xavier D, et al. (2011) RIVAL Tria Group. Radial versus femoral access for coronary angiography and intervention in patients with acute coronary syndromes (RIVAL): a randomised, parallel group, multicentre trial. Lancet 377: 1409-1420.

19. Romagnoli E, Biondi-Zoccai G, Sciahbasi A, Politi L, Rigattieri S, et al. (2012) Radial versus femoral randomized investigation in ST-segment elevation acute coronary syndrome: The RIFLE-STEACS (Radial Versus Femoral Randomized Investigation in ST-Elevation Acute Coronary Syndrome) study. J Am Coll Cardiol 60: 2481-2489.

20. Achenbach S, Ropers D, Kallert L, Turan N, Krahner R, et al. (2008) Transradial versus transfemoral approach for coronary angiography and intervention in patients above 75 years of age. Catheter Cardiovasc Interv 72: 629-635.

21. Bertrand OF, Bagur R, Costerousse O, Rodes-Cabau J (2010) Transradial vs femoral percutaneous coronary intervention for left main disease in octogenarians. Indian Heart J 62: 234-237.

22. Jimenez V, Colin E, Ortiz A, Miguel A, Bastos G (2011) Feasibility and predictors of success and outcome. TCT Abstract/Poster/Vascular Access and closure.

23. Hu F, Yang Y, Qiao S, Xu B, Liu H, et al. (2012) Comparison between radial and femoral approach for percuatnous coronary intervention in patients aged 80 years or older. J Interv cardiology 25: 513-517.

24. Jaffe R, Hong T, Sharieff W, Chisholm RJ, Kutryk MJ, et al. (2007) Comparison of radial versus femoral approach for percutaneous coronary interventions in octogenarians. Catheter Cardiovasc Interv 69: 815-820.

25. Jin C, Xu Y, Qiao SB, Tang XR, Wu YJ, et al. (2017) Transradial versus transfemoral approach for percutaneous coronary intervention in elderly patients in China: A retrospective analysis. Chin Med Sci J 32: 161-170.

26. Klinke WP, Hilton JD, Warburton RN, Warburton WP, Tan RP (2004) Comparison of treatment outcomes in patients $>$ or $=80$ years undergoing transradial versus transfemoral coronary intervention. Am J Cardiol 93: 12821285.

27. Koutouzis M, Matejka G, Olivecrona G, Grip L, Albertsson P (2010) Radia vs. femoral approach for primary percutaneous coronary intervention in octogenarians. Cardiovasc Revasc Med 11: 79-83.

28. Louvard Y, Benamer H, Garot P, Hildick-Smith D, Loubeyre C, et al. (2004) Comparison of transradial and transfemoral approaches for coronary angiography and angioplasty in octogenarians (The Octoplus study). Am J Cardiol 94: 1177-1180.

29. Secco GG, Marinucci L, Uguccioni L, Parisi R, Uguccioni S, et al. (2013) Transradial versus transfemoral approach for primary percutaneous coronary interventions in elderly patients. J Invasive Cardiol 25: 254-256.

30. Tammam K, Ikari Y, Yoshimachi F, Saito F, Hassan W (2017) Impact of transradial coronary intervention on bleeding complications in octogenarians. Cardiovasc Interv Ther 32: 18-23

31. Yan ZX, Zhou YJ, Zhao YX, Liu YY, Shi DM, et al. (2008) Safety and feasibility of transradial approach for primary percutaneous coronary intervention in elderly patients with acute myocardial infarction. Chin Med J (Engl) 121: 782-786.

32. Ziakas A, Gomma A, McDonald J, Klinke P, Hilton D (2007) A comparison of the radial and the femoral approaches in primary or rescue percutaneous coronary intervention for acute myocardial infarction in the elderly. Acute Card Care 9: 93-96.

33. Anjum I, Khan MA, Aadil M, Faraz A, Farooqui M, et al. Transradial vs. Transfemoral Approach in Cardiac Catheterization: A Literature Review. Cureus 9: e1309.

34. Roussanov O, Wilson SJ, Henley K, Estacio G, Hill J, et al. (2007) Costeffectiveness of the radial versus femoral artery approach to diagnostic cardiac catheterization. J Invasive Cardiol 19: 349-353.

35. Aamir S, Mohammed S, Sudhir R (2016) Transradial approach for coronary procedures in the elderly population. J Geriatr Cardiol 13: 798-806. 